\title{
Factors That Influence the Prescription of Antipsychotics for Patients with Schizophrenia in China
}

\author{
Tian-Mei Si ${ }^{1}$, Liang Shu ${ }^{1}, \mathrm{Ke}-\mathrm{Qing} \mathrm{Li}^{2}$, Xie-He Liư ${ }^{3}, \mathrm{Qi}-\mathrm{Yi} \mathrm{Mei}^{4}$, Gao-Hua Wang ${ }^{5}$, Pei-Shen Bai ${ }^{7}$, Li-Ping $\mathrm{Ji}^{6}$, \\ Xian-Sheng Chen ${ }^{8}$, Cui $\mathrm{Ma}^{9}$, Jian-Guo Shi ${ }^{10}$, Hong-Yan Zhang ${ }^{1}$, Hong Ma ${ }^{1}$, Xin $\mathrm{Yu}^{1}$ \\ 'Key Laboratory of Mental Health, Institute of Mental Health, Peking University, Beijing, ${ }^{2}$ Hebei Mental Health Center, Baoding, ${ }^{3}$ Mental \\ Health Center of Huaxi Hospital, Sichuan University, Chengdu, ${ }^{4}$ Guangii Hospital, Suzhou, ${ }^{5}$ Department of Mental Health. People's Hospital \\ of Wuhan, Wuchang, ${ }^{6} \mathrm{Jilin}$ Neuro-Psychiatric Hospital, Siping, ${ }^{7}$ The First Hospital of Shanxi Medical University, Taiyuan, ${ }^{8}$ Jiangxi Mental \\ Health Hospital, Nanchang, ${ }^{9}$ Guangzhou Mental Health Hospital, Guangzhou, ${ }^{10}$ Xi'An Mental Health Center, Xi'An, China
}

Objective: To investigate the patterns of antipsychotic use in China and to analyze the factors that influence antipsychotic prescriptions.

Methods: A standardized survey was conducted from May 20 to 242002 in five different regions of China with varying economic levels. The patterns of antipsychotic medication use were analyzed in a sample of 4,779 patients with schizophrenia. The survey gathered information on demographic characteristics, clinical profiles, and antipsychotic medications prescribed. Multiple logistic regression was used to analyze factors related to patterns of antipsychotic medication use.

Results: A plurality of patients with schizophrenia was treated with clozapine (39\%); this was followed by risperidone, sulpride, chlorpromazine, perphenazine, and haloperidol. More than $56.3 \%$ of patients were treated with only one atypical antipsychotic. The mean daily dose of chlorpromazine was $365 \pm 253 \mathrm{mg}$ (mean \pm standard deviation), and $6.5 \%$ of patients were treated with depot injections of typical antipsychotic medications. A total of $73.7 \%(n=3,523)$ of patients with schizophrenia received monotherapy, $24.8 \%(n=1,183)$ received two antipsychotics, $1.1 \%(n=52)$ received three antipsychotics, and one received four different antipsychotics. Patients often simultaneously received other classes of medications including anticholinergic agents, benzodiazepines, $\beta$-blockers, antidepressants, and mood stabilizers. Economic status and clinical symptoms were the main factors that contributed to the patterns of antipsychotic prescription.

Conclusion: The present study suggests that atypical antipsychotic medications, especially clozapine, are the primary psychiatric treatments of choice in the management of schizophrenia in China. Moreover, the economic status and clinical profile of the patient are the major factors affecting the prescription of antipsychotic medication.

KEY WORDS: Schizophrenia; Antipsychotic drugs; Prescriptions; China; Survey; Clozapine.

\section{INTRODUCTION}

Schizophrenia, a chronic mental disorder characterized by the disintegration of thought processes and emotional responsiveness, has a life-time prevalence of $0.655 \%$ in the Chinese population. ${ }^{1)}$ It most commonly manifests as a broad range of abnormal psychological and behavioral processes including hallucinations, psychotic ideation, delusions, disorganized thought processes, inappropriate

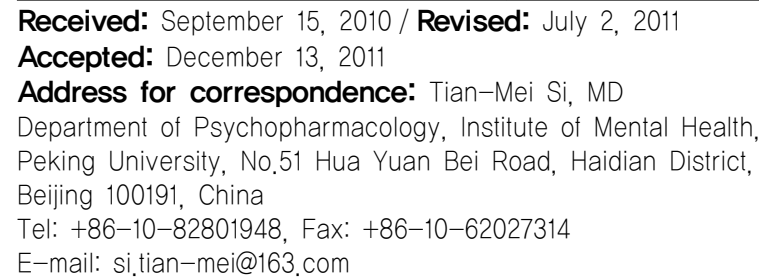

affect, and impaired ability to form and implement intentions and to engage in planning; this condition is often accompanied by significant social dysfunction. Patients frequently alternate between acute psychotic episodes and stable periods with residual symptoms or partial remission. A significant number of patients with schizophrenia ultimately experience chronic disability. Indeed, one-third of homeless adults suffer from severe mental illnesses, including schizophrenia. Schizophrenia has great economic costs, including the direct costs of health care as well as the costs associated with loss of productivity and provision of caregivers. The economic burden, including the use of social and medical resources, ${ }^{2-11)}$ placed by schizophrenia on Chinese society, families, and individuals is astounding.

Pharmacotherapy ${ }^{5,8,9)}$ is the primary psychiatric treat- 
ment for schizophrenia, especially for the care of acute psychotic episodes, the improvement of inter-episode symptoms, and the prevention of future episodes. Antipsychotic medications are divided into two classes: first-generation (also known as conventional or typical) ${ }^{2)}$ antipsychotics and second-generation (also known as atypical) antipsychotics. Typical antipsychotics act primarily as central dopamine $\mathrm{D}_{2}$-receptor antagonists. Typical antipsychotics include chlorpromazine, haloperidol, perphenazine, and sulpride. Atypical antipsychotics have a greater affinity for serotonin-2A $\left(5-\mathrm{HT}_{2 \mathrm{~A}}\right)$ receptors than to $\mathrm{D}_{2}$ receptors. Atypical antipsychotics include clozapine, risperidone, ${ }^{4)}$ olanzapine, and quetiapine. Clozapine was the first atypical antipsychotic to be developed in the 1970s. Clozapine is very effective in the treatment of both the positive and negative symptoms associated with schizophrenia. Moreover, it produces fewer extrapyramidal side effects (EPS), including tardive dyskinesia, and has less effect on serum prolactin compared to typical antipsychotic medications. The risk of inducing agranulocytosis with clozapine limited its use in many countries. In addition to the high risk of agranulocytosis, clozapine has also been associated with an increased incidence of sudden death, myocardiopathy, weight gain, diabetes mellitus, and lipid abnormalities. ${ }^{7)}$ In 1988, however, clozapine was demonstrated to be effective in the treatment of refractory schizophrenia in a major multicenter clinical trial. ${ }^{3)}$ As a result, clozapine was approved by the Food and Drug Administration (FDA) and reintroduced for the treatment of resistant schizophrenia in 1990. Clozapine, however, has consistently been used in China since 1975, and it remains one of the most frequently prescribed psychiatric medications throughout this country due to its established efficacy, limited side effects, and minimal cost. ${ }^{5,6)}$

The pharmacological treatment of choice for schizophrenia is typical and atypical antipsychotics, including clozapine, risperidone, olanzapine, and quetiapine. In addition to the differences in the pharmacological properties $^{3)}$ and clinical profiles of the two classes of medications, a significant difference in the cost of typical and atypical medications also exists. Indeed, atypical antipsychotics can cost over 40 times more than typical antipsychotics.

Regions in China vary tremendously with regard to geography, economics, race, culture, and customs as well as in mental health resources. Previous studies have reported that the patterns of antipsychotic use differ across regions. ${ }^{5,68,9)}$ The Chinese Psychopharmacology Algorithm
Project (CPAP) was established in 1999. The CPAP is a set of guidelines for clinical psychopharmacotherapy in China. An investigation into the general patterns of antipsychotic prescription in China would aid in the formulation of CPAP guidelines as well as contribute additional data to the State Health Care Programme. Therefore, the Ministry of Health in China and the Peking University Institute of Mental Health supported the present study of the patterns of antipsychotic medication use in patients with schizophrenia in different regions in China characterized by varying economic levels.

\section{METHODS}

\section{Data Source}

The data for the present study were derived from a nationwide investigation of antipsychotic prescription medications. According to the Gross Domestic Product (GDP) in $2000,{ }^{10)}$ Chinese provinces can be separated into five different economic regions. Ten provinces were selected for use in the present study, and one city was chosen from each selected province. The cities selected for this study included Beijing, Suzhou, and Guangzhou (level I: highest economic level); Baoding (level II); Siping and Wuhan (level III); Taiyuan (level IV); and Xi'An, Nanchang, and Chengdu (level V: lowest economic level). Within each selected city, mental health hospitals were divided into three categories according to academic level and daily number of outpatients and inpatients. One or more hospitals from each category were chosen for the present study.

\section{Samples}

The sample for the present study included all outpatients and inpatients who met the criteria for schizophrenia included in the International Classification of Diseases-Tenth Revision (ICD-10) and who were treated at the selected hospitals during May 20-24, 2002. This multi-center investigation was conducted during a 1-week period.

\section{Survey Instruments}

All subjects in the present study completed a standard questionnaire. Data on demographic and clinical characteristics, physical condition, illness duration, significant symptoms during the previous month, illness severity, illness type, and dosage and adverse effects of prescribed antipsychotics were collected for all patients. Antipsychotic medications were converted into chlorpromazine-equivalent milligrams as a standardized unit of 
dosage. $^{11,12)}$

\section{Statistical Analysis}

Statistical analyses of the data were conducted with SPSS 13.0 (SPSS Inc., Chicago, IL, USA). Chi-square and one-way ANOVA analyses were utilized. Multinomial regression models were used to analyze the factors affecting the patterns of prescription of typical antipsychotics, clozapine, and non-clozapine atypical antipsychotics (i.e., risperidone, olanzapine, and quetiapine) across different regions and economic levels. Due to the high rate of clozapine prescription in China, clozapine and non-clozapine medications were analyzed separately as dependent variables. More than 20 independent variables, including patient demographic characteristics and clinical symptoms, were included in the logistic regression models of the patterns of antipsychotic prescription. Only those variables that were found to have a significant impact on the dependent variables are discussed.

\section{RESULTS}

\section{Demographic Characteristics}

A total of 4,779 patients with schizophrenia were surveyed in the present study $(1,079,804,970,502$, and 1,424 subjects in each of the five economic regions, respectively). The demographic characteristics are presented in Table 1. The mean age of the patients in the present study was $34.5 \pm 12.8$ years old (mean \pm standard deviation [SD], median of 32 years of age). The monthly family income was $497 \pm 523$ Yuan per person (median of 400 RenMinBi); $46.2 \%$ of the patients were female, and $21.6 \%$ of the patients $(n=1,030)$ reported a positive family history for schizophrenia. Significant differences across the five regions were noted in the mean age, duration of illness, family income, and sex of patients.

A total of $41.2 \%$ of the subjects in the present study were outpatients $(n=1,969)$, and $58.8 \%(n=2,810)$ were inpatients. At the time of the study, 45.9\% $(1,290 / 2,810)$ of the subjects were experiencing their first hospital ad- mission for schizophrenia. Inpatient subjects were significantly older, had a longer illness, and reported more severe symptoms compared to outpatient subjects (Table 2).

\section{Patterns of Antipsychotic Use}

Type and frequency of antipsychotics prescribed

Nearly all (99.6\%) the subjects in the present study were treated with antipsychotic medications. The types of antipsychotics used by the subjects are presented in Table 3. Overall, clozapine was the most frequently prescribed medication, accounting for $40 \%$ of all prescriptions $(42 \%$ of inpatient subjects and $34.6 \%$ of outpatient subjects, respectively). Additionally, $23.2 \%$ of the participants were treated with risperidone $(20.3 \%$ and $27.3 \%$ of inpatient and outpatient subjects, respectively), $18.1 \%$ were treated with sulpride, $16.5 \%$ were treated with chlorpromazine, $12.4 \%$ were treated with perphenazine, and 5.6\% were treated with haloperidol. Significant differences were noted in the frequency of prescriptions for certain medications across different regions and economic levels. The mean chlorpromazine-equivalent daily dose was $365 \pm 253$ $\mathrm{mg}$ (mean $\pm \mathrm{SD}$; range: $12.5-4,125 \mathrm{mg}$ ). The daily dose of medication was significantly higher among inpatient than outpatient subjects $(409 \pm 274 \mathrm{mg}$ vs. $300 \pm 201 \mathrm{mg}, F=223$, $p<0.000)$, and $6.5 \%$ of patients $(\mathrm{n}=312)$ used depot injections of antipsychotics (152 inpatient and 160 outpatient subjects). Subjects receiving depot injections were significantly older (mean age of 37 vs. 34 years, $F=15.0$,

Table 2. Demographic and clinical symptoms of outpatients and inpatients

\begin{tabular}{lccc}
\hline & $\begin{array}{c}\text { Inpatients } \\
(\mathrm{n}=1,969)\end{array}$ & $\begin{array}{c}\text { Outpatients } \\
(\mathrm{n}=2,810)\end{array}$ & F or $x^{2}$ \\
\hline Age (year) & $35.46 \pm 13.01$ & $33.02 \pm 12.26$ & $42.9^{* *}$ \\
Duration of illness (month) & $92.53 \pm 113.7$ & $60.99 \pm 83.00$ & $109.7^{* *}$ \\
$\begin{array}{l}\text { Family income } \\
\text { (RMB/month/person) }\end{array}$ & $492 \pm 434$ & $504 \pm 626$ & 0.532 \\
Gender, \% female & 50.2 & 42.8 & $26.68^{* *}$ \\
\hline
\end{tabular}

n, number; RMB, RenMinBi.

${ }^{* *} p<0.001$ among five level regions.

Data are presented as mean \pm standard deviation.

Table 1. Demographic and clinical symptoms of patients in five level regions

\begin{tabular}{|c|c|c|c|c|c|c|c|}
\hline & I $(n=1,079)$ & $\|(n=804)$ & III $(n=970)$ & IV $(n=502)$ & $V(n=1,424)$ & Total $(n=4,779)$ & F or $\chi^{2}$ \\
\hline Age (year) & $39.1 \pm 13.3$ & $33.4 \pm 11.9$ & $31.0 \pm 10.8$ & $32.6 \pm 12.3$ & $34.6 \pm 13.2$ & $34.5 \pm 12.8$ & $58.95^{* *}$ \\
\hline Duration of illness (month) & $123.2 \pm 128.6$ & $72.7 \pm 90.5$ & $43.9 \pm 65.5$ & $62.5 \pm 82.8$ & $80.3 \pm 104.3$ & $79.5 \pm 103.3$ & $86.29^{* *}$ \\
\hline Family income (RMB/month/person) & $826 \pm 760$ & $323 \pm 341$ & $412 \pm 428$ & $366 \pm 465$ & $461 \pm 336$ & $497 \pm 523$ & $157.2^{* *}$ \\
\hline Gender, \% female & 45.7 & 51.1 & 48.7 & 46.2 & 41.1 & 46.2 & $26.57^{\star *}$ \\
\hline
\end{tabular}

number: RMB, RenMinBi

${ }^{* *} p<0.001$ among five level regions.

Data are presented as mean \pm standard deviation. 
Table 3. Type and frequency of prescribed antipsychotics in the treatment of schizophrenia

\begin{tabular}{|c|c|c|c|c|c|c|c|}
\hline & I $(n=1,079)$ & $\|(n=804)$ & III $(n=970)$ & IV $(n=502)$ & $V(n=1,424)$ & Total $(n=4,779)$ & F or $\chi^{2}$ \\
\hline Clozapine & $343(31.8)$ & $353(43.9)$ & $418(43.1)$ & 145 (28.9) & $604(42.4)$ & $1,863(39.0)$ & $67.13^{* *}$ \\
\hline Risperidone & $314(29.1)$ & $120(14.9)$ & $304(31.3)$ & 111 (22.2) & $259(18.2)$ & $1,108(23.2)$ & $108.5^{\star \star}$ \\
\hline Chlorpromazine & $218(20.2)$ & $114(14.2)$ & $132(13.6)$ & $100(19.9)$ & $225(15.8)$ & $789(16.5)$ & $24.53^{\star *}$ \\
\hline Sulpride & $137(12.7)$ & $156(19.4)$ & $146(15.1)$ & $94(18.7)$ & $330(23.2)$ & $863(18.1)$ & $53.21^{* *}$ \\
\hline Perphenazine & $119(11.0)$ & $114(14.2)$ & $98(10.1)$ & $73(14.5)$ & $186(13.1)$ & $590(12.4)$ & $11.6^{*}$ \\
\hline Haloperidol & $52(4.8)$ & $80(10.0)$ & $24(2.5)$ & $24(4.8)$ & $86(6.0)$ & $266(5.6)$ & $49.38^{* *}$ \\
\hline Atypicals $^{\dagger}$ (non-clozapine) & $380(35.2)$ & $147(18.3)$ & $325(33.5)$ & $111(22.1)$ & $295(20.7)$ & $1,258(26.3)$ & $124.3^{* \star}$ \\
\hline Typicals & $552(51.2)$ & $439(54.6)$ & $414(42.7)$ & $318(63.3)$ & $842(59.1)$ & $2,565(53.7)$ & $86.10^{* *}$ \\
\hline Dosage $^{\ddagger}$ (mg/day) & $388 \pm 282$ & $364 \pm 286$ & $327 \pm 182$ & $333 \pm 297$ & $383 \pm 229$ & $364 \pm 253$ & $11.56^{* *}$ \\
\hline
\end{tabular}

n, number.

${ }^{*} p<0.05 .{ }^{* *} p<0.001$. ${ }^{\dagger}$ Including risperidone, olanzapine and quetiapine. ${ }^{\dagger}$ Chlorpromazine equivalent dosage.

Data are presented as $n(\%)$ or mean \pm standard deviation.

$p<0.001)$ and had a significantly longer illness duration (122 vs. 76 months, $F=57.3, p<0.001$ ) compared to subjects not receiving depot injections. Moreover, those treated with depot injections were more likely to be inpatients $\left(\chi^{2}=7.8, p=0.005\right)$ and to reside in level- $\mathrm{V}$ regions $(8.0 \%$, $\left.\chi^{2}=15.1, p<0.005\right)$. In the present study, $53.7 \%$ of the subjects used typical antipsychotics and $65.3 \%$ of subjects used atypical antipsychotics.

Pharmacological polytherapy and concomitant medication

In the present study, 3,523 (73.7\%) of the patients received pharmacological monotherapy, 1,183 (24.8\%) were treated with two different medications, 52 (1.1\%) received a combination of three medications, and one was treated with four medications. Significant differences were noted in the frequency of pharmacological polytherapy across the different regions ( $\left.\chi^{2}=16.9, p=0.002\right)$. The most frequent combination consisted of clozapine with another typical or atypical antipsychotic medication. Patients who received pharmacological polytherapy reported more severe symptoms compared to patients treated with pharmacological monotherapy $\left(\chi^{2}=7.76, p=\right.$ $0.005)$. Polytherapy with other classes of medications included anticholinergics $(n=1,432,30.0 \%)$, benzodiazepines $(n=951,19.9 \%), \beta$-blockers $(n=475,10.0 \%)$, antidepressants (4.8\%), and mood stabilizers (4.8\%) (Table 4).

\section{Risk Factors Associated with the Use of Antipsychotic Medication}

According to multivariate regression analysis, several patient characteristics, including age, illness duration, clinical syndromes, and family income, were correlated with the use of clozapine, non-clozapine atypical medications, or typical medications (Table 5). Patients with less family income and longer illnesses as well as those who experienced hallucinations and delusions or displayed impaired social and professional functioning were
Table 4. Polypharmacy and concomitant medication

\begin{tabular}{lrr}
\hline & Number $(n)$ & $\%$ \\
\hline Mono-pharmacy $(n=3,523)$ & 1,536 & 43.6 \\
Typical medications & 894 & 25.4 \\
Non-clozapine atypical medications & 1,093 & 31.0 \\
Clozapine & & \\
Polypharmacy $(n=1,236)$ & 620 & 13.0 \\
Clozapine+typical medications & 159 & 3.3 \\
Clozapine+non-clozapine atypical medications & 187 & 3.9 \\
Typicals+non-clozapine atypical medications & 320 & 6.7 \\
Typicals+typicals medications & & \\
Concomitant medications & 1,432 & 30.0 \\
Anticholinergics & 951 & 19.9 \\
Benzodiazepines & 475 & 10.0 \\
$\beta$-blocker & 228 & 4.8 \\
Antidepressants & 228 & 4.8 \\
Mood stabilizers & & \\
\hline
\end{tabular}

significantly more likely to use typical antipsychotic medications (odds ratio $[\mathrm{OR}]=0.59,1.012,0.793$, and 1.165 , respectively). Patients with more family income and shorter illnesses as well as those who did not experience hallucinations and delusions, were younger, or had comorbid affective syndromes (i.e., depression or mania) were significantly more likely to use non-clozapine atypical antipsychotic medications $(\mathrm{OR}=3.828,0.969,1.874$, 1.363 , and 0.985 , respectively). Non-clozapine atypical medications included risperidone, olanzapine, and quetiapine. Patients who experienced aggression or disorganized speech and behaviors were significantly less likely to use non-clozapine atypical medications $(\mathrm{OR}=0.824$ and 0.719 , respectively). Clozapine is the prototypical atypical antipsychotic, and its cost is approximately the same as that of typical antipsychotics in China. Patients with less family income and longer illnesses and those who experienced aggression or negative symptoms were significantly more likely to use clozapine $(\mathrm{OR}=0.594$, $1.010,1.271,1.536$, and 1.306 respectively). Patients with long illnesses and those who experienced negative symptoms were the most likely to be treated with depot injections ( $\mathrm{OR}=1.002$ and 1.489 , respectively). The results 
Table 5. Factors influencing the likelihood of using typical medications, clozapine and non-clozapine atypical medications

\begin{tabular}{|c|c|c|c|c|}
\hline Factors & B & $\begin{array}{l}\text { Jan Wald, } \\
\text { square value }\end{array}$ & Significance level & $\begin{array}{l}\text { Expectation } \\
\text { (B) }\end{array}$ \\
\hline \multicolumn{5}{|l|}{ Typical medications } \\
\hline Family income & -.527 & 48.449 & .000 & .590 \\
\hline Duration of illness & .012 & 17.242 & .000 & 1.012 \\
\hline Hallucination and delusion & -.232 & 11.962 & .001 & .793 \\
\hline Impaired social and professional function & .153 & 3.975 & .046 & 1.165 \\
\hline \multicolumn{5}{|l|}{ Non-clozapine atypical medications } \\
\hline Age & -.015 & 21.389 & .000 & .985 \\
\hline Family income & 1.342 & 214.582 & .000 & 3.828 \\
\hline Duration of illness & -.032 & 36.963 & .000 & .969 \\
\hline Hallucination and delusion & .628 & 53.215 & .000 & 1.874 \\
\hline Disorganized speech and behaviors & -.193 & 6.855 & .009 & .824 \\
\hline Comorbid with affection syndromes & .310 & 6.623 & .010 & 1.363 \\
\hline Aggression & -.330 & 12.684 & .000 & .719 \\
\hline \multicolumn{5}{|l|}{ Clozapine } \\
\hline Family income & -.522 & 39.090 & .000 & .594 \\
\hline Duration of illness & .010 & 11.006 & .001 & 1.010 \\
\hline Hallucination and delusion & -.156 & 5.040 & .025 & .855 \\
\hline Negative syndromes & .240 & 13.928 & .000 & 1.271 \\
\hline Depression mood & -.872 & 19.051 & .000 & .418 \\
\hline Disorganized speech and behaviors & .429 & 6.155 & .013 & 1.536 \\
\hline Aggression & .267 & 12.155 & .000 & 1.306 \\
\hline \multicolumn{5}{|l|}{ Depot } \\
\hline Duration of illness & .002 & 29.886 & .000 & 1.002 \\
\hline Negative symptoms & .398 & 10.753 & .001 & 1.489 \\
\hline
\end{tabular}

B, coefficient of regression.

Table 6. Factors affecting the likelihood of using polypharmacy and concomitant therapy

\begin{tabular}{|c|c|c|c|c|}
\hline Factors & B & $\begin{array}{l}\text { Jan Wald, } \\
\text { square value }\end{array}$ & Significance level & $\begin{array}{l}\text { Expectation } \\
\text { (B) }\end{array}$ \\
\hline \multicolumn{5}{|l|}{ Polypharmacy } \\
\hline Negative symptoms & .473 & 46.764 & .000 & 1.605 \\
\hline Comorbid with affective symptoms & .364 & 9.693 & .002 & 1.439 \\
\hline Impaired social or occupational functions & .217 & 5.656 & .017 & 1.242 \\
\hline \multicolumn{5}{|l|}{ Concomitant anticholinergics } \\
\hline $\begin{array}{l}\text { EPS, e.g., tremor, dyskinesia, akathisia } \\
\beta \text {-blockers }\end{array}$ & 1.477 & 232.461 & .000 & 4.379 \\
\hline Autonomic system reaction & 0.782 & 4.531 & 0.033 & 2.185 \\
\hline \multicolumn{5}{|l|}{ Benzodiazepines } \\
\hline Excitement & .389 & 10.067 & .002 & 1.475 \\
\hline Depressive mood & 1.018 & 37.653 & .000 & 2.768 \\
\hline Retardation or rigidity & .484 & 6.296 & .012 & 1.622 \\
\hline Aggression & 0.526 & 25.530 & .000 & 1.691 \\
\hline \multicolumn{5}{|l|}{ Mood stabilizers } \\
\hline Comorbid with manic symptoms & 1.530 & 85.835 & 0.000 & 4.617 \\
\hline \multicolumn{5}{|l|}{ Antidepressants } \\
\hline Depressive mood & 0.471 & 5.488 & 0.019 & 1.601 \\
\hline
\end{tabular}

B, coefficient of regression; EPS, extrapyramidal side effects.

of the present study suggest that the clinical symptoms and the economic status of patients are the most important factors contributing to the selection of antipsychotic medications used for the treatment of schizophrenia.

\section{Risk Factors Associated with Polypharmacy and Concomitant Antipsychotics}

More than $25 \%$ of the patients in the present study were treated with pharmacological polytherapy. This may be attributable to several factors, including the frequency of negative symptoms, comorbid mood symptoms, and social and occupational dysfunction in this patient population. The use of concomitant anticholinergics, $\beta$-blockers, benzodiazepines, antidepressants, and mood stabilizers was significantly associated with the clinical characteristics of the patient (e.g., EPS, excitement, retardation, rigidity or aggression, and affective symptoms) (Table 6).

\section{Adverse Effects}

According to the patients, the most common adverse effects associated with antipsychotic treatment were akathisia $(14.5 \%)$, tremors $(14.5 \%)$, somnolence $(13.2 \%)$, dyskinesia (9.4\%), dystonia (3.7\%, including tardive dyskinesia), constipation (22.6\%), salivation (17.9\%), dry mouth $(14.6 \%)$, postural hypotension $(3.8 \%)$, changes in body weight $(15.3 \%)$, amenorrhea $(2.8 \%)$, sexual dys- 
function (1.8\%), changes in white blood cell (WBC) count $(3.1 \%)$, liver dysfunction (3.0\%), glucose abnormalities $(0.4 \%)$, and lipid abnormalities $(0.9 \%)$. Multivariate regression analysis showed that EPS were associated with treatment with typical antipsychotic medication. Both typical and atypical antipsychotics, especially clozapine, were associated with adverse effects on the autonomic nervous system.

\section{DISCUSSION}

To our knowledge, the present study was the first investigation of the pattern of antipsychotic medication use in hospitals across different regions and economic levels in China. The present results reflect the unique profile of pharmacotherapy for patients with schizophrenia and the pattern of antipsychotic prescription in China compared with that in other countries. ${ }^{13-15)}$

The median age of the subjects in the present study was 32 years, and age was correlated with the clinical features of the disease in the current study. Nearly $70 \%$ of patients with schizophrenia respond positively to pharmacotherapy during their first-episode, ${ }^{16)}$ but they are often at increased risk of relapse due to poor treatment compliance. ${ }^{17-19)}$ Previous studies have shown that approximately $80 \%$ of first-episode patients experienced a second episode within 5 years of the first episode. ${ }^{20)}$ Repeat episodes of schizophrenia are frequently associated with worse patient outcomes and residual symptoms, leading to longer periods of treatment or hospitalization. Indeed, inpatient subjects were older and had longer illnesses compared to outpatient subjects in the present study. The family income of patients in the current study was not equal to that according to the GDP level of the general population in China. ${ }^{10)}$ The economic status of patients with schizophrenia may decline as the illness continues over time. A total of $79.9 \%$ of the patients experienced impaired social and occupational functioning. Previous investigations have shown economic status to be one of the main factors prompting patients to seek treatment, especially in less affluent areas. ${ }^{21-30)}$ Significant differences in demographic characteristics were noted. These differences among the five regions may be related to differences in local culture, customs, and economic status.

The most commonly prescribed antipsychotic medication for the treatment of schizophrenia in China was clozapine. Specifically, $42 \%$ of inpatient subjects were treated with clozapine in the present study (in monotherapy or in combination with other antipsychotic medi- cations). Clozapine was the treatment of choice for several reasons including its therapeutic effects, its cost, and the longer illnesses experienced by the patients. Atypical antipsychotic medications, including clozapine, were prescribed more frequently than typical antipsychotics as a monotherapy in China (56.4\% for atypical medications vs. $43.6 \%$ for typical medications). These results are consistent with reports from populations in the United States. ${ }^{13)}$ The mean chlorpromazine-equivalent dose in the present study was similar to that reported in a study from Hong Kong. ${ }^{21)}$ Those treated with depot injections of antipsychotics $(6.5 \%)$ were typically outpatients with a chronic mental illness. Depot injections ensure compliance in patients with chronic illnesses. ${ }^{22)}$ Differences among the five regions in the use of antipsychotic medications may be related to differences in the demographic characteristic of the patients.

Schizophrenia is a disease that involves severe disability. The use of atypical antipsychotics in the treatment of schizophrenia has significantly increased the cost of treating this illness. ${ }^{23-25)}$ The present survey demonstrated that family economic status and the clinical characteristics of the patient are important factors in the prescription of antipsychotic medications. Less family income was related to impaired social and occupational functioning and to a chronic course of the illness. Patients with these clinical features preferred less costly medications (i.e., typical antipsychotics or clozapine). Patients who experienced hallucinations, delusions, depression, or mania were more likely to use atypical antipsychotics. Patients who exhibited disorganized speech or behaviors or aggression or negative symptoms were more likely to be treated with clozapine. These results suggest that atypical antipsychotics target a wider range of symptoms in patients with schizophrenia, which is also supported by the psychopharmacological findings of these medications. ${ }^{26,27)}$ Recent reports have suggested that atypical antipsychotic medications were more effective than typical antipsychotics in the treatment of schizophrenia. ${ }^{28)}$

The benefits and risks of pharmacological polytherapy warrant further investigation. ${ }^{29)}$ It appears that polytherapy may be beneficial to some individuals, especially those with chronic or refractory schizophrenia. Pharmacological polytherapy, however, increases the risk of EPS as well as the economic burden on the patient. The present study revealed that less than $25 \%$ of patients in China received polytherapy. The most common treatment involved a combination of clozapine and another atypical or typical antipsychotic medication. Moreover, this study 
demonstrated that the most common concomitant medications were anticholinergics, $\beta$-blockers, benzodiazepines, antidepressants, and mood stabilizers. These medications were typically prescribed to relieve the adverse effects of the antipsychotic drugs and the comorbid symptoms, including excitement, rigidity, insomnia, depression, and mania. The most frequent adverse effects of antipsychotics reported in the present study were EPS and effects on the autonomic nervous system.

The present study revealed that nearly all of the patients with schizophrenia in China were treated with antipsychotic medications. The demographic and clinical characteristics of patients contributed to the patterns of antipsychotic medication use across regions in China. The results of this study will be helpful in the development of novel scientific treatments for patients with schizophrenia.

\section{REFERENCES}

1. Chen $\mathrm{CH}$, Shen $\mathrm{Y}$, Zhang WX, Li SR, Huang YQ, Wang $\mathrm{JR}$, et al. Epidemiological Survey on schizophrenia in 7 areas of China. Chin J Psychiatry 1998;31:72-74.

2. World Congress of Psychiatry. Consensus statement on the use and usefulness of second generation antipsychotic medication. Yokohama: World Congress of Psychiatry; 2002.

3. Kane J, Honigfeld G, Singer J, Meltzer H. Clozapine for the treatment-resistant schizophrenic. A double-blind comparison with chlorpromazine. Arch Gen Psychiatry 1988;45:789796.

4. Chakos M, Lieberman J, Hoffman E, Bradford D, Sheitman B. Effectiveness of second-generation antipsychotics in patients with treatment-resistant schizophrenia: a review and meta-analysis of randomized trials. Am J Psychiatry 2001; 158:518-526.

5. Fang $\mathrm{M}, \mathrm{Hu} \mathrm{SH}$, Wang Q. Investigaiton of psychotropic drugs therapy in hospitalized patients. Chengdu Med J 2002; 28:139-140.

6. Zhao JY, Shi YZ, Zhao XX, Lv LX, Guo SQ. Survey on psychotropic drug used in first-episode schizophrenics. China J Health Psychol 2002;10:167-168.

7. Remington G, Khramov I. Health care utilization in patients with schizophrenia maintained on atypical versus conventional antipsychotics. Prog Neuropsychopharmacol Biol Psychiatry 2001;25:363-369.

8. Li YL, Mao RH, Ni YX, Lian DY. Relation between utilization of antipsychotic drug and therapeutical compliance in schizophrenic patients. Chin Gen Practice 2002;5:358-359.

9. Wu PL. Use of antipsychotics among chronic schizophrenic inpatients. Med Guide 2001;20:452-453.

10. Liu H. China Statistical Yearbook. Beijing: China Statistics Press; 1999. p.49-108.

11. Kane JM. Schizophrenia. N Engl J Med 1996;334:34-41.

12. Taylor R, McConnell D, McConnell H, Abel K, Kerwin R.
The Bethlem and Maudsley NHS Trust 1999 prescribing guidelines. 5th ed. London: Martin Dunitz; 1999.

13. Side Effects of Antipsychotic Medications: Physician's Choice of Medication and Patient Compliance. Dallas, Texas, California. January 22, 1999. Proceedings of a roundtable. J Clin Psychiatry 2000;61 Suppl 8:3-66.

14. Lyu RR, McCombs JS, Johnstone BM, Muse DN. Use of conventional antipsychotics and the cost of treating schizophrenia. Health Care Financ Rev 2001;23:83-99.

15. McCombs JS, Nichol MB, Stimmel GL, Shi J, Smith RR. Use patterns for antipsychotic medications in medicaid patients with schizophrenia. J Clin Psychiatry 1999;60 Suppl 19:5-11

16. Lieberman J, Jody D, Geisler S, Alvir J, Loebel A, Szymanski S, et al. Time course and biologic correlates of treatment response in first-episode schizophrenia. Arch Gen Psychiatry 1993;50:369-376.

17. Aquila R, Weiden PJ, Emanuel M. Compliance and the rehabilitation alliance. J Clin Psychiatry 1999;60 Suppl 19: 23-27.

18. Eder U, Koller D, Hummer M, Kurzthaler I, Ulmer H, Fleischhacker WW. Variables influencing outpatients compliance. Schizophr Res 1998;29:161.

19. Linden M, Godermann F. Compliance in long-term treatment of schizophrenia. Schizophr Res 1998;29:161-162.

20. Prudo R, Blum HM. Five-year outcome and prognosis in schizophrenia: a report from the London Field Research Centre of the International Pilot Study of Schizophrenia. Br J Psychiatry 1987;150:345-354.

21. Chow LY, Ungvari GS, Chiu H, Leung T. Psychotropic drug prescription for chronic patients in a long-stay care facility. Hong Kong J Psychiatry 1999;9:15-20.

22. Kane JM, Aguglia E, Altamura AC, Ayuso Gutierrez JL, Brunello N, Fleischhacker WW, et al. Guidelines for depot antipsychotic treatment in schizophrenia. European Neuropsychopharmacology Consensus Conference in Siena, Italy. Eur Neuropsychopharmacol 1998;8:55-66.

23. Knapp M, Kavanagh S. Economic outcomes and costs in the treatment of schizophrenia. Clin Ther 1997;19:128-138.

24. Martin A, Leslie D. Psychiatric inpatient, outpatient, and medication utilization and costs among privately insured youths, 1997-2000. Am J Psychiatry 2003;160:757-764.

25. Revicki DA. Pharmacoeconomic studies of atypical antipsychotic drugs for the treatment of schizophrenia. Schizophr Res 1999;35 Suppl:S101-S109.

26. Ereshefsky L. Pharmacologic and pharmacokinetic considerations in choosing an antipsychotic. J Clin Psychiatry 1999; 60 Suppl 10:20-30.

27. Stahl SM. Selecting an atypical antipsychotic by combining clinical experience with guidelines from clinical trials. $J$ Clin Psychiatry 1999;60 Suppl 10:31-41.

28. Csernansky JG, Schuchart EK. Relapse and rehospitalisation rates in patients with schizophrenia: effects of second generation antipsychotics. CNS Drugs 2002;16:473-484.

29. Stahl SM. Antipsychotic polypharmacy: squandering precious resources? J Clin Psychiatry 2002;63:93-94.

30. Murali V, Oyebode F. Poverty, social inequality and mental health. Advances in Psychiatric Treatment 2004;10:216-224. 